\title{
Capacity Analysis of Cooperative Relaying Networks with Adaptive Relaying Scheme Selection
}

\author{
Kunihiko TESHIMA $^{\dagger a)}$, Student Member, Koji YAMAMOTO ${ }^{\dagger \dagger}{ }^{\dagger}$, Hidekazu MURATA $^{\dagger}$, Members $^{\dagger}$, \\ and Susumu YOSHIDA ${ }^{\dagger}$, Fellow
}

\begin{abstract}
SUMMARY In the present paper, the performance of cooperative relaying networks with adaptive relaying scheme selection is analyzed. Cooperative relaying is a new technique to achieve spatial diversity gain by using neighboring stations. However, when multiple stations transmit simultaneously, the number of interference signals increases. Therefore, the introduction of cooperative relaying in radio communication systems does not always increase the network capacity due to the co-channel interference. Therefore, in order to achieve high spectral efficiency, it is necessary to select cooperative relaying or non-cooperative relaying adaptively. Assuming both centralized and decentralized adaptive controls, the spectrum efficiency is evaluated. The performance under decentralized control is evaluated using a game-theoretic approach. Simulation results show that the introduction of cooperative relaying with centralized control always increases the spectral efficiency. On the other hand, Simulation results also show that, when each source selects a relaying scheme independently and selfishly to maximize its own spectral efficiency, the introduction of the cooperative relaying may reduce the spectral efficiency due to the increase in the number of interference signals.

key words: cooperative relaying, multihop, spectral efficiency, adaptive control, game theory
\end{abstract}

\section{Introduction}

Cooperative relaying is a new technique to enhance the spectral efficiency of multihop radio networks [1]-[6]. In cooperative relaying, the source, destination, and relay stations share resources to achieve spatial diversity gain against fading or spatial multiplexing gain. Thus, the performance of cooperative relaying has primarily been evaluated in terms of error rate or spectral efficiency, assuming a single-isolated communication [4]-[6] or two pairs of communications [1], [3]. The introduction of cooperative relaying has been reported to reduce error rate and enhance spectral efficiency.

However, cooperative relaying does not always improve the spectrum efficiency when co-channel interference exists [7]. This is because, in some cooperative relaying, multiple stations transmit simultaneously to achieve diversity gain. Such cooperative relaying inherently increases the number of co-channel interference signals compared to noncooperative transmission and may reduce the received signal to interference plus noise power ratio (SINR) when the interference has a significant impact on the received SINR.

Manuscript received October 9, 2008.

Manuscript revised March 23, 2009.

${ }^{\dagger}$ The authors are with the Graduate School of Informatics, Kyoto University, Kyoto-shi, 606-8501 Japan.

${ }^{\dagger}$ The author is with Wireless@KTH, Royal Institute of Technology, Stockholm, 16440 Sweden.

a) E-mail: contact-h21@ @anase.kuee.kyoto-u.ac.jp

DOI: 10.1587/transcom.E92.B.3744
In order to achieve high spectral efficiency, it is necessary to select cooperative relaying or non-cooperative relaying adaptively. The centralized scheduling algorithm for cooperative relaying to improve the throughput is proposed in [8]. However, when each source selects a relaying scheme independently and selfishly to maximize its own spectral efficiency, it is unclear whether the network capacity increases.

A promising approach to analyzing the decentralized control and selfishness is game theory, which deals with how individuals interact and compete for resources. Game theoretic approach is also used for power control in code division multiple access data networks [9]. The effect of "selfish" random access in aloha systems has been evaluated using game-theoretic analysis [10].

In the present paper, the impact of the introduction of cooperative relaying with the adaptive relaying scheme selection (ARSS) is analyzed. First, we evaluate the outage bandwidth efficiency (BE) of each relaying scheme, i.e., cooperative relaying, non-cooperative two-hop transmission, and single-hop transmission. BE is defined to be the maximum end-to-end transmission rate through multiple hops per unit bandwidth. Second, assuming centralized control, we evaluate the network capacity with optimal scheduling by exhaustive search. Finally, we analyze the performance under decentralized control in which each source independently selects a relaying scheme and route in order to enhance its own BE. We report a game-theoretic analysis of a decentralized adaptive relaying scheme selection (DARSS) problem.

The remainder of the present paper is organized as follows. In Sect. 2, we define the system model. In Sect. 3, we evaluate the outage $\mathrm{BE}$ in interference-limited environments and compare the outage $\mathrm{BE}$ with the ergodic $\mathrm{BE}$ discussed in [7]. In Sect. 4, we introduce the game theory and define the DARSS game. In Sect. 5, we discuss the impact of ARSS. In Sect. 6, we conclude the present paper.

\section{System Model}

Consider a cooperative relaying system with only one relay station $(\mathrm{R})$ located between a given source $(\mathrm{S})$ and destination (D), as illustrated in Fig. 1(a). Each station is equipped with an omnidirectional antenna and is assumed to be able to either transmit or receive at a given time. 


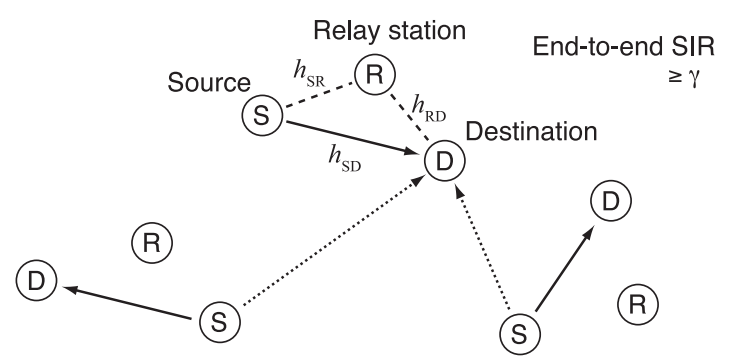

(a) Model of relay channels.

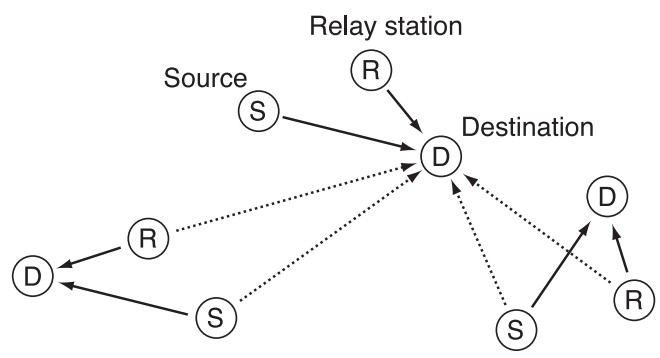

(b) The number of interference signals is doubled due to simultaneous transmission by the source and the relay station when using cooperative relaying (RD, SR) and $(\mathrm{R}, \mathrm{SR})$ in slot 2 .

Fig. 1 Solid arrows represent desired signals and dashed arrows represent co-channel interference [7].

\subsection{Cooperative Relaying}

One technique to enable forwarding a signal is dividing the spectrum into different orthogonal time slots and allocating these slots to each transmitting station [4]. Time slots are assumed not to be synchronized among all routes.

The relay station is assumed to decode and forward the signal received from the source to the destination using two time slots and let $i(=1,2)$ denote the index of the slot. We assume the following three cooperative schemes (RD, SR), $(\mathrm{RD}, \mathrm{R})$, and $(\mathrm{R}, \mathrm{SR})$ and two non-cooperative schemes, as shown in Fig. 2, where for example (RD, SR) denotes that both $R$ and $D$ receive in slot 1 and both $S$ and $R$ transmit in slot 2 .

(RD, SR) This scheme is proposed in [4]. In slot 1, both the relay station and the destination receive the signal from the source. In slot 2, both the source and the relay station transmit to the destination.

$(\mathbf{R D}, \mathbf{R})$ This scheme is proposed in [3], [11]. In slot 1, both the relay station and the destination receive the signal from the source. In slot 2 , only the relay station transmits to the destination.

(R, SR) This scheme is also proposed in [3], [11]. In slot 1 , only the relay station receives the signal from the source. In slot 2, both the source and the relay station transmit to the destination.

Single-hop In slots 1 and 2, the source transmits directly to the destination.

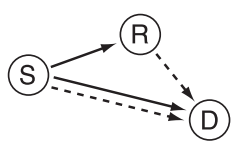

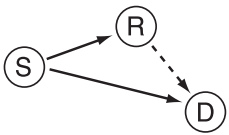

(b) $(\mathrm{RD}, \mathrm{R})$.

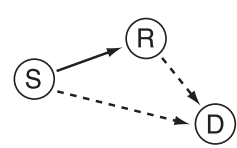

(c) $(\mathrm{R}, \mathrm{SR})$ (a) $(\mathrm{RD}, \mathrm{SR})$
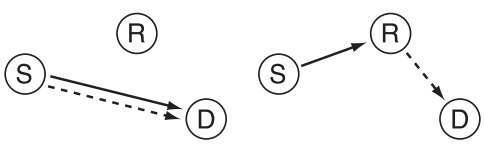

(e) Two-hop $=(\mathrm{R}, \mathrm{R})$

Fig. 2 Three cooperative and two non-cooperative schemes. Solid arrows represent transmissions in slot 1 , and dashed arrows represent transmissions in slot 2 [7]

Two-hop In slot 1 , only the relay station receives the signal from the source. In slot 2, only the relay station transmits to the destination.

\subsection{Bandwidth Efficiency}

\subsubsection{Cooperative Relaying (RD, SR)}

The signal transmitted by the source in slot $i$ is denoted as $x_{i}$. All interference signals are assumed to be equivalent to Gaussian noise over the channel, as in [12] (which is discussed in more detail in [13]). The signals received by the relay station in slot $1, y_{\mathrm{R}, 1}$, and received by the destination in slot $i, y_{\mathrm{D}, i}$, are then given by

$$
\begin{aligned}
& y_{\mathrm{R}, 1}=\sqrt{E_{\mathrm{SR}}} h_{\mathrm{SR}} x_{1}+n_{\mathrm{R}, 1} \\
& y_{\mathrm{D}, 1}=\sqrt{E_{\mathrm{SD}}} h_{\mathrm{SD}} x_{1}+n_{\mathrm{D}, 1} \\
& y_{\mathrm{D}, 2}=\sqrt{E_{\mathrm{RD}}} h_{\mathrm{RD}} x_{1}+\sqrt{E_{\mathrm{SD}}} h_{\mathrm{SD}} x_{2}+n_{\mathrm{D}, 2}
\end{aligned}
$$

where $E_{\mathrm{XY}}$ denotes the average signal energy at the station $\mathrm{Y}$ through $\mathrm{X}-\mathrm{Y}$ link, and $h_{\mathrm{XY}}$ denotes the complex unitpower channel gain of X-Y link, and $n_{\mathrm{X}, i} \sim \mathcal{C N}\left(0, N_{\mathrm{X}, i}\right)$ denotes the noise plus interference treated as complex Gaussian noise processes at the station X. Here, $\mathcal{C N}\left(0, \sigma^{2}\right)$ is a complex Gaussian random variable with mean zero and variance $\sigma^{2}$.

Let $N$ denote the noise power, and let $\lambda_{i}=N / N_{\mathrm{D}, i}$, $\mu=E_{\mathrm{SR}} / E_{\mathrm{SD}}$, and $v=E_{\mathrm{RD}} / E_{\mathrm{SD}}$. In the following, we assume a frequency-flat block fading channel. In addition, we assume that the relay station has the perfect information of $h_{\mathrm{SR}}$ and that the destination has the perfect information of $h_{\mathrm{SD}}$ and $h_{\mathrm{RD}}$. Ergodic $\mathrm{BE} f_{(\mathrm{RD}, \mathrm{SR})}$ can be expressed as

$$
\begin{aligned}
& f_{(\mathrm{RD}, \mathrm{SR})}=\frac{1}{2} \min \left\{\mathrm{E}\left[R_{1,2}^{\max }\right], \mathrm{E}\left[R_{\text {relay }}^{\max }+R_{2}^{\max }\right]\right\} \\
& R_{1,2}^{\max } \triangleq \log _{2} \operatorname{det}\left(\boldsymbol{I}_{2}+\boldsymbol{A} \boldsymbol{A}^{\mathrm{H}} \frac{E_{\mathrm{SD}}}{N}\right) \\
& R_{\text {relay }}^{\max } \triangleq \log _{2}\left(1+\frac{E_{\mathrm{SR}}\left|h_{\mathrm{SR}}\right|^{2}}{N_{\mathrm{R}, 1}}\right) \\
& R_{2}^{\max } \triangleq \log _{2}\left(1+\frac{E_{\mathrm{SD}}\left|h_{\mathrm{SD}}\right|^{2}}{N_{\mathrm{D}, 2}}\right)
\end{aligned}
$$




$$
\boldsymbol{A}=\left[\begin{array}{cc}
\sqrt{\lambda_{1}} h_{\mathrm{SD}} & 0 \\
\sqrt{\lambda_{2} v} h_{\mathrm{RD}} & \sqrt{\lambda_{2}} h_{\mathrm{SD}}
\end{array}\right]
$$

where $\boldsymbol{I}_{2}$ denotes the identity matrix of size 2 and $E[\cdot]$ denotes the expectation operator (refer to [4], [7] for more details).

\subsubsection{Cooperative Relaying ( $\mathrm{RD}, \mathrm{R})$}

Ergodic $\mathrm{BE} f_{(\mathrm{RD}, \mathrm{R})}$ can be expressed as

$$
\begin{aligned}
& f_{(\mathrm{RD}, \mathrm{R})}=\frac{1}{2} \min \left\{\mathrm{E}\left[R_{\text {relay }}^{\max }\right], \mathrm{E}\left[R_{1}^{\max }\right]\right\} \\
& R_{1}^{\max } \triangleq \log _{2}\left(1+\|\boldsymbol{a}\|^{2} \frac{E_{\mathrm{SD}}}{N}\right)
\end{aligned}
$$

where $\boldsymbol{a}$ denotes the first column of $\boldsymbol{A}$ and $\|\cdot\|$ is the Euclidean norm [4], [7].

\subsubsection{Cooperative Relaying (R, SR)}

The ergodic $\mathrm{BE} f_{(\mathrm{R}, \mathrm{SR})}$ can be expressed as

$$
\begin{aligned}
& f_{(\mathrm{R}, \mathrm{SR})}=\frac{1}{2} \min \left\{\mathrm{E}\left[R_{1}{ }^{\max \prime}\right], \mathrm{E}\left[R_{\text {relay }}^{\max }+R_{2}^{\max }\right]\right\} \\
& R_{1}{ }^{\max } \triangleq \log _{2}\left(1+\|\boldsymbol{b}\|^{2} \frac{E_{\mathrm{SD}}}{N}\right)
\end{aligned}
$$

where $\boldsymbol{b}^{\mathrm{T}}$ is the second row of $\boldsymbol{A}$.

\subsubsection{Single-Hop Transmission}

Ergodic BE $f_{1}$ can be expressed as follows [7].

$$
f_{1}=\mathrm{E}\left[\log _{2}\left(1+\frac{E_{\mathrm{SD}}\left|h_{\mathrm{SD}}\right|^{2}}{N_{\mathrm{D}, i}}\right)\right] \text {. }
$$

\subsubsection{Two-Hop Transmission}

The ergodic BE of two-hop $f_{2}$ is given by

$$
\begin{aligned}
& f_{2}=\frac{1}{2} \min \left\{\mathrm{E}\left[R_{\text {relay }}^{\max }\right], \mathrm{E}\left[R_{2}{ }^{\max \prime}\right]\right\} \\
& R_{2}{ }^{{ }^{\max }} \triangleq \log _{2}\left(1+\frac{E_{\mathrm{RD}}\left|h_{\mathrm{RD}}\right|^{2}}{N_{\mathrm{D}, 2}}\right) .
\end{aligned}
$$

\subsection{Outage Bandwidth Efficiency}

We define the $p \%$ outage $\mathrm{BE} C_{p}^{\text {out }}$ as the BE guaranteed for $(100-p) \%$ of the channel realizations. Then, $C_{p}^{\text {out }}$ is given by

$$
F\left(C_{p}^{\text {out }}\right)=p \%
$$

where $F(\cdot)$ denotes the cumulative distribution function of the instantaneous BE. The instantaneous transmission rate $R_{(\mathrm{RD}, \mathrm{SR})}, R_{(\mathrm{RD}, \mathrm{R})}, R_{(\mathrm{R}, \mathrm{SR})}, R_{1}$, and $R_{2}$ must satisfy

$$
R_{(\mathrm{RD}, \mathrm{SR})}<\frac{1}{2} \min \left\{R_{1,2}^{\max }, R_{\text {relay }}^{\max }+R_{2}^{\max }\right\}
$$

$$
\begin{aligned}
& R_{(\mathrm{RD}, \mathrm{R})}<\frac{1}{2} \min \left\{R_{\text {relay }}^{\max }, R_{1}^{\max }\right\} \\
& R_{(\mathrm{R}, \mathrm{SR})}<\frac{1}{2} \min \left\{R_{1}{ }^{\max \prime}, R_{\text {relay }}^{\max }+R_{2}^{\max }\right\} \\
& R_{1}<\log _{2}\left(1+\frac{E_{\mathrm{SD}}\left|h_{\mathrm{SD}}\right|^{2}}{N_{\mathrm{D}, i}}\right) \\
& R_{2}<\frac{1}{2} \min \left\{R_{\text {relay }}^{\max }, R_{2}{ }^{\max \prime}\right\}
\end{aligned}
$$

where the right-hand sides of (17), (18), (19), (20), and (21) are the instantaneous BE of each scheme.

\section{Bandwidth Efficiency of Individual Cooperative Re- laying in Interference-Limited Environments}

Before evaluating the impact of ARSS, we evaluate the ergodic and outage BE of individual cooperative relaying. Thus, in this section, we assume that all communication has the same relaying scheme for all transmissions. In addition, we assume interference-limied environments for theoretical analysis.

\subsection{Interference-Limited Environments}

In this section, we assume interference-limited environments in which co-channel interference dominates the thermal noise. In order to represent the density of the simultaneous communication, we introduce the end-to-end signal to interference ratio (ESIR), $\gamma$, as follows. Consider the situation in which every source transmits directly to a designated destination regardless of whether each source-destination pair can communicate directly, as shown in Fig. 1(a), and we designate the received signal to interference ratio (SIR) as the ESIR. This ESIR is considered to be an appropriate parameter to reflect the density of the simultaneous communication since the absolute value of this density does not have meaning in the following simple model (which is discussed in more detail in [14]).

The spectral efficiency of cooperative relaying is sensitive to several factors, particularly fairness [15], [16]. Since the purpose of this section is to examine the impact of cooperative relaying on the spectral efficiency of wireless networks, we set aside the fairness problem for now. Thus, we investigate the spectral efficiency under the condition that all communication at a given time has almost the same condition, i.e., communication methods for all transmissions are the same and ESIR is larger than $\gamma$. In addition, the interference power from the source and that from the designated relay station are assumed to be the same. Under this assumption, the interference power is proportional to the number of transmitters in a route, as described in Sect. 3.2.

Let $I_{i}$ denote the interference power in slot $i$, rather than the identity matrix. Since we assume interference-limited environments, $N_{\mathrm{R}, 1}$ and $N_{\mathrm{D}, 1}$ are replaced with $I_{1}$, and $N_{\mathrm{D}, 2}$ are replaced with $I_{2}$. In addition, let $I$ denote the interference power when every source transmits directly to a designated destination and let us redefine $\lambda_{i}=I / I_{i}$. Then the ESIR can 
Table 1 Ratio of interference power $\lambda_{1}, \lambda_{2}$.

\begin{tabular}{c|c|c}
\hline & 1, two-hop, (RD, R) & $(\mathrm{RD}, \mathrm{SR}),(\mathrm{R}, \mathrm{SR})$ \\
\hline$\lambda_{1}$ & 1 & $2 / 3$ \\
$\lambda_{2}$ & 1 & $2 / 3$ \\
\hline
\end{tabular}

Table 2 Parameters used in the numerical evaluation.

\begin{tabular}{c|c}
\hline Parameters & Values \\
\hline Path loss exponent $\alpha$ & 2 \\
Channel model & Rayleigh fading \\
Area environment & Interference-limited \\
\hline
\end{tabular}

be written as $\gamma=E_{\mathrm{SD}} / I$ and we obtain

$$
\begin{aligned}
& R_{1,2}^{\max }=\log _{2} \operatorname{det}\left(\boldsymbol{I}_{2}+\boldsymbol{A} \boldsymbol{A}^{\mathrm{H}} \gamma\right) \\
& R_{\mathrm{relay}}^{\max }=\log _{2}\left(1+\left|h_{\mathrm{SR}}\right|^{2} \mu \lambda_{1} \gamma\right) \\
& R_{2}^{\max }=\log _{2}\left(1+\left|h_{\mathrm{SD}}\right|^{2} \lambda_{2} \gamma\right) \\
& R_{1}^{\max }=\log _{2}\left(1+\|\boldsymbol{a}\|^{2} \gamma\right) \\
& R_{1}{ }^{\max \prime}=\log _{2}\left(1+\|\boldsymbol{b}\|^{2} \gamma\right) \\
& \log _{2}\left(1+\frac{E_{\mathrm{SD}}\left|h_{\mathrm{SD}}\right|^{2}}{N_{\mathrm{D}, i}}\right)=\log _{2}\left(1+\left|h_{\mathrm{SD}}\right|^{2} \gamma\right) \\
& R_{2}{ }^{{ }^{\max }}=\log _{2}\left(1+\left|h_{\mathrm{RD}}\right|^{2} \nu \lambda_{2} \gamma\right)
\end{aligned}
$$

as a function of ESIR as follows as in [7]. In this section, we evaluate the BE using the ESIR as a parameter.

\subsection{Number of Interference Signals}

There are two types of transmission scheme in terms of the number of transmitters in a route. When using schemes ( RD, SR) or (R, SR) both the source and the relay station transmit in slot 2, as shown in Fig. 1(b), resulting in doubled interference signals compared to other situations, in which either the source or the relay station transmit during a given time.

We then consider the resultant SIR when using schemes (RD, SR) or (R, SR). When assuming different transmissions are not synchronized, on average, in half of the routes, both the source and the relay station transmit simultaneously, and in the other half of the routes, either the source alone or the relay station alone transmits. Therefore, the number of interference signals is increased by a factor of $3 / 2$, and the received SIR in the $(R D, S R)$ and $(R, S R)$ is approximately $2 / 3$ of that in the other schemes. Table 1 summarizes the ratio of interference power $\lambda_{i}$ for each scheme.

\subsection{Assumption}

Table 2 summarizes the parameters used in the following evaluation. The complex channel gains $h_{\mathrm{SD}}, h_{\mathrm{SR}}$, and $h_{\mathrm{RD}}$ are assumed to be i.i.d. $C \mathcal{N}(0,1)$, i.e., Rayleigh fading. We assume path loss with the path loss exponent $\alpha$. For the sake of simplicity, the effect of shadowing is not considered. Since the interference-limited situation is assumed, the absolute value of transmit power does not affect the received

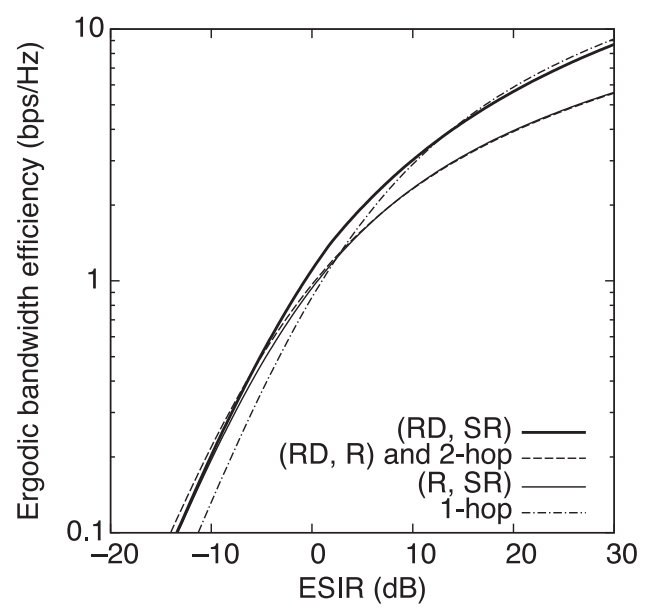

(a) Ergodic bandwidth efficiency.

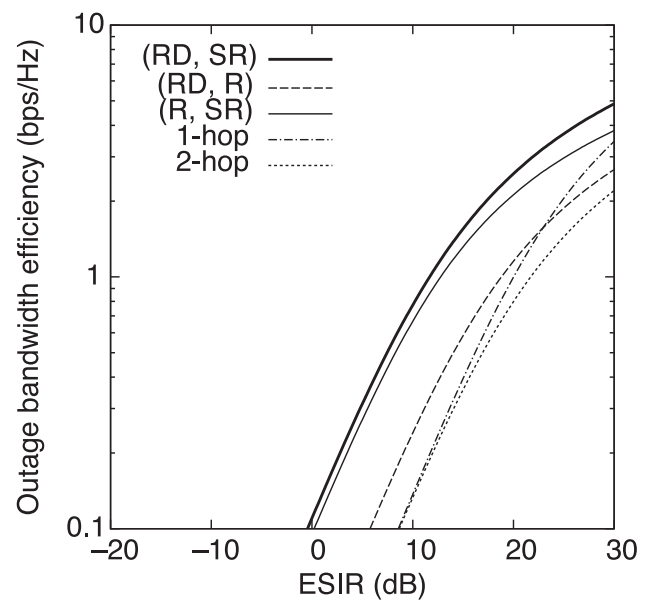

(b) Outage bandwidth efficiency.

Fig.3 Bandwidth efficiency in interference-limited environments.

SIR. Thus, the transmit power is assumed to be the same for all stations. Note that it is not fair to compare these schemes in terms of power consumption.

The relay station is assumed to be on the center point of a straight line segment from the source to the destination. Under this assumption, $\mu=v=2^{\alpha}$. While these assumptions are not necessarily realistic, they do enable us to gain insight into the design of cooperative relaying systems.

\subsection{Numerical Results}

First, for comparison, Fig. 3(a) shows the ergodic BE discussed in [7]. The technique used to achieve the highest ergodic BE is changed from single-hop transmission to the (RD, SR) scheme and then to two-hop transmission and the $(\mathrm{RD}, \mathrm{R})$ scheme along with the decrease of ESIR. This result shows that the advantage of cooperative relaying in interference-limited environments depends on the density of the simultaneous transmission [7]. Therefore, in order to maximize the ergodic BE, it is necessary to select the relaying scheme adaptively.

Second, Fig. 3(b) shows the $1 \%$ outage BE. In contrast 
to the ergodic $\mathrm{BE}$, the (RD, SR) scheme always outperforms the other two cooperative relaying schemes and two-hop transmission. The reason is as follows. In $(\mathrm{RD}, \mathrm{R})$ and two-hop transmission, the source transmits the signal only in slot 1 , and the relay station needs to be able to decode this signal. Thus, the transmission rate depends heavily on the channel gain of S-R link. On the other hand, in (RD, $\mathrm{SR})$, the relay station does not need to be able to decode the signal transmitted by the source in slot 2 . Thus, the transmission rate in slot 2 depends only on the channel gain of S-D link. Therefore, (RD, SR) achieves high diversity gain as compared with (RD, R). The performance of the outage $\mathrm{BE}$ is sensitive to the reduction of received power due to fading. Therefore, even though the simultaneous transmission of both the source and the relay station increases the number of interference signals, the (RD, SR) scheme is superior to the other two cooperative relaying schemes and two-hop transmission. This relationship does not depend on $p$, while the ESIR in which single-hop transmission outperforms the other schemes decreases with the increase of $p$.

\section{Game-Theoretic Framework}

Game theory is a promising approach for analyzing the performance of cooperative relaying networks under the decentralized control. In this section, the game theory is explained and the DARSS game is defined.

\subsection{Normal Form Game}

A game in normal form has three aspects. First, there is a set of players, $I$, which we take to be the finite set $\{1,2, \ldots, I\}$. Second, there is a set of pure strategies, $S_{i}(i \in \mathcal{I})$, that is available to each player. Finally, there is a set of payoff functions, $u_{i}$. Each player has a preference for an outcome. The payoff function represents the player's preference and maps the player's anticipated outcome given his or her selected strategy to a real number. In the game, each player's objective is to select a strategy, $s_{i} \in S_{i}$, so as to maximize his or her own payoff function. A mixed strategy for player $i$ is a probability distribution over pure strategies $S_{i}$. In the present paper, unless otherwise noted, we assume pure strategies because mixed strategies do not seem reasonable in studies of stability (similar arguments are presented in [17], [18]).

A fundamental solution concept in game theory is a Nash equilibrium, which is a point at which neither player gains by changing his or her strategy unilaterally, so that neither player has an incentive to deviate [19]. A pure strategy profile, $s^{*}=\left(s_{1}{ }^{*}, \ldots, s_{I}{ }^{*}\right)$, is a Nash equilibrium if, for all players $i$,

$$
\begin{aligned}
u_{i}\left(s^{*}\right) \geq u_{i}\left(s_{1}{ }^{*}, \ldots, s_{i-1}{ }^{*}, s_{i}, s_{i+1}{ }^{*}, \ldots,\right. & \left.s_{I}{ }^{*}\right) \\
& \forall s_{i} \in S_{i} .
\end{aligned}
$$

In particular, for a two-player game, a pure strategy profile is a Nash equilibrium if

$$
\begin{array}{ll}
u_{1}\left(s_{1}{ }^{*}, s_{2}{ }^{*}\right) \geq u_{1}\left(s_{1}, s_{2}{ }^{*}\right) & \forall s_{1} \in S_{1} \\
u_{2}\left(s_{1}{ }^{*}, s_{2}{ }^{*}\right) \geq u_{2}\left(s_{1}{ }^{*}, s_{2}\right) & \forall s_{2} \in S_{2} .
\end{array}
$$

\subsection{Decentralized Adaptive Relaying Scheme Selection Game}

In order to evaluate the impact of ARSS under decentralized control, we define the DARSS game. In DARSS game, sources are assumed to be players. Each player has to select the relaying scheme and one relay station so that the maximum BE is achieved. As a relaying scheme, we consider the cooperative relaying, two-hop transmission, single-hop transmission, and stop transmission. Thus we use the ergodic BE (Eqs. (4), (9), (11), (13), and (14)) and instantaneous BE (the right-hand sides of (17)-(21)) as payoff functions. To evaluate the performance of three cases in which (RD, SR), (RD, R), or (R, SR) are employed as a cooperative relaying scheme, we consider the following four cases.

case $_{(\text {no coop.) }}$ Each source can select the non-cooperative schemes, that is, two-hop transmission, single-hop transmission, and stop transmission.

case $_{(\mathbf{R D}, \text { SR) }}$ Each source can select the (RD, SR) scheme and the non-cooperative schemes.

case $_{(\mathbf{R D}, \mathbf{R})}$ Each source can select the (RD, R) scheme and the non-cooperative schemes.

case $_{(\mathbf{R}, \text { SR) }}$ Each source can select the (R, SR) scheme and the non-cooperative schemes.

Each source is assumed to be able to estimate the BE of all sources as a consequence of their selection. In addition, the control overhead associated with ARSS is not taken into account. This is a reasonable assumption because the purpose of the present paper is to analyze the achievable capacity due to the ARSS.

\section{Analysis of Adaptive Relaying Scheme Selection}

In Sect. 3, we assumed a simple model in which all communication at a given time has almost the same condition, and evaluated the ergodic and outage BE of individual cooperative relaying in interference-limied environments. In this section, assuming the network in which all stations are uniformly and independently distributed, we evaluate the impact of the ARSS in cooperative relaying networks.

In order to evaluate the impact of the ARSS, we evaluate the maximum total $\mathrm{BE}$ under the centralized control with perfect knowledge (hereinafter referred to as "network capacity"), and the total BE under equilibrium conditions (hereinafter referred to as "equilibrium BE"). Total BE is defined as the BE summed over all simultaneous transmitting sources in the network. This equilibrium BE is a reasonable payoff as a result of the DARSS game [20]. 


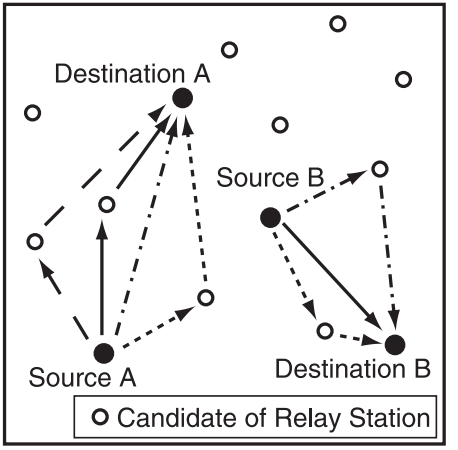

Fig. 4 Example network. Each source selects one relaying scheme and one relay station.

\subsection{Assumption}

For the sake of simplicity, we consider a cooperative relaying network consisting of two source-destination pairs and ten relay candidates as has been done in [20]. This is a reasonable assumption because the purpose of the present paper is to evaluate the impact of decentralized adaptive controls by multiple decision-making entities on the achievable throughput in cooperative relaying networks compared to centralized controls. All stations are uniformly and independently distributed in a square area and are stationary throughout the following evaluation. Under this assumption, Nash equilibria can be calculated through (30). Since the purpose of this analysis is to evaluate the equilibrium BE, Nash equilibrium is calculated by exhaustive search. In the simulations, the results are averaged over 2400 topologies.

The received power is assumed to decay in proportion to the square of the distance between the stations. Then, we define $P$ and $D$ as the reference transmit power and the reference source-destination distance in which the received signal to noise ratio is calculated to be $0 \mathrm{~dB}$. We assume a $5 D \times 5 D$ square area and that all stations transmit at the same power level $P^{\prime}$. The complex channel gains $h_{\mathrm{SD}}, h_{\mathrm{SR}}$, and $h_{\mathrm{RD}}$ are assumed to be i.i.d. $C \mathcal{N}(0,1)$, i.e., Rayleigh fading. For the sake of simplicity, the effect of shadowing is not considered.

Time slots are assumed not to be synchronized among all stations in the network. When using cooperative relaying or two-hop transmission, there are two transmission states due to two slots, that is, the time slots of two sources may or may not be the same. For the sake of simplicity, we assume that these two states have equal probability.

Figure 4 shows an example network. In the DARSS game, source A selects one relaying scheme and must also select one relay station, so as to maximize its own BE. Similarly, source B selects its own strategy.

\subsection{Analysis}

Figure 5 shows the percentage of three categories based on

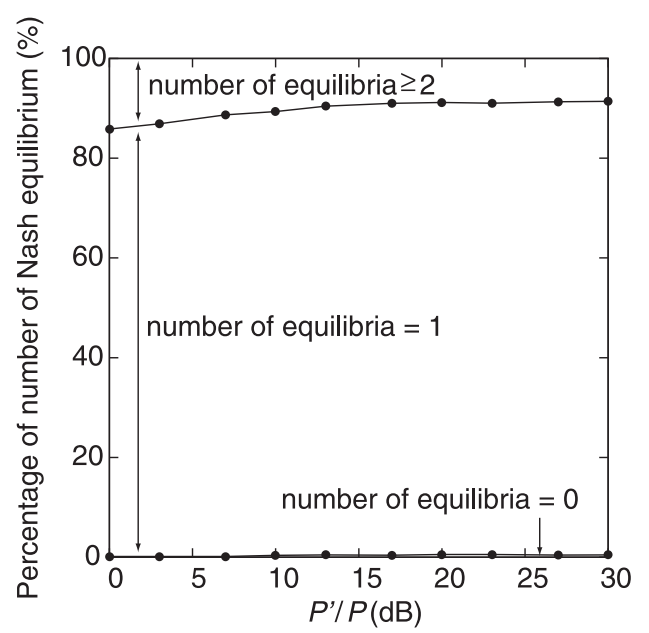

Fig. 5 Percentage of the number of equilibria of case $_{(\mathrm{RD}, \mathrm{SR})}$.

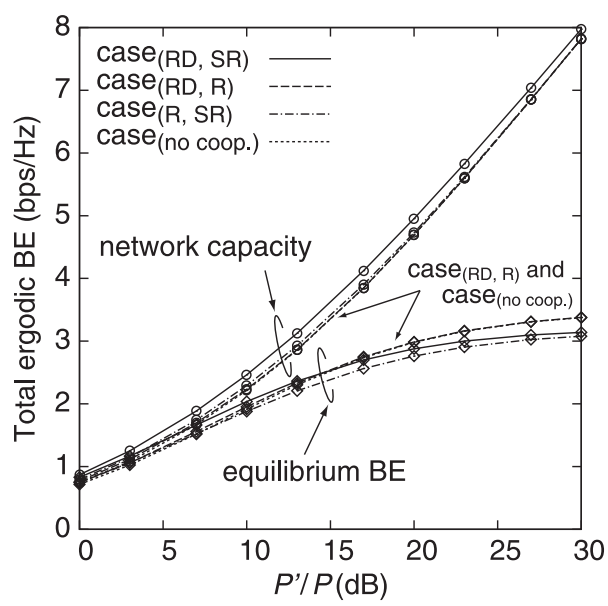

Fig. 6 Transmit power dependence of total ergodic BE. Here, case $_{(\mathrm{RD}, \mathrm{R})}$

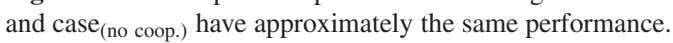

the number of Nash equilibria of case $_{(\mathrm{RD}, \mathrm{SR})}$. The DARSS game has one or more Nash equilibria in most cases. A similar finding applies to case ${ }_{(\mathrm{RD}, \mathrm{R})}$, $\operatorname{case}_{(\mathrm{R}, \mathrm{SR})}$, and case $\mathrm{c}_{(\text {no coop.) }}$. Therefore, in the following analysis, we evaluate the performance of networks in which there is at least one Nash equilibrium.

Figure 6 shows the network capacity for the networks with at least one Nash equilibrium, and Fig. 7 shows the selected strategies of case ${ }_{(\mathrm{RD}, \mathrm{SR})}$. Here, the symbol $[\mathrm{X}, \mathrm{Y}]$ in Fig. 7, e.g., [coop., single], represents that $\mathrm{X}$ and $\mathrm{Y}$ are the strategies selected by sources. As shown in Fig. 6, the introduction of cooperative relaying always increases the network capacity. Then, the strategies selected by sources depend on the transmit power, as shown in Fig. 7(a). When the transmit power is low, both sources transmit. On the other hand, when the transmit power is high, only one source transmits.

Figure 6 also shows the equilibrium BE for the networks with at least one Nash equilibrium. When the transmit power is low, the DARSS game attains the BE near the 


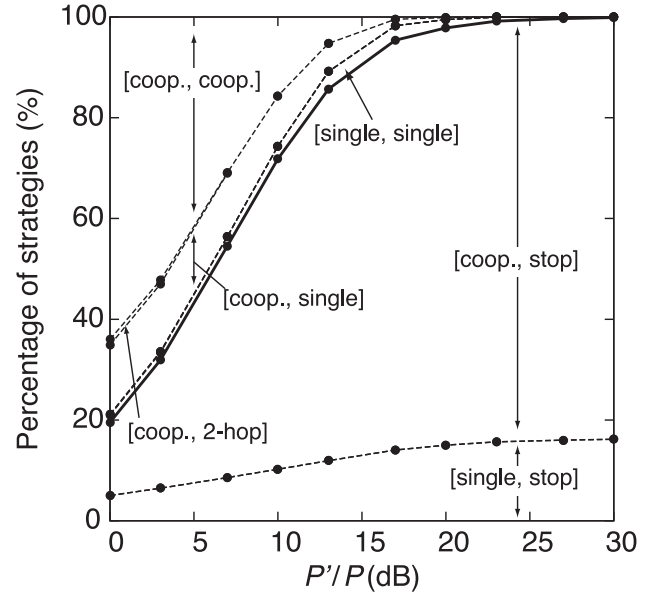

(a) Under centralized scheduling.

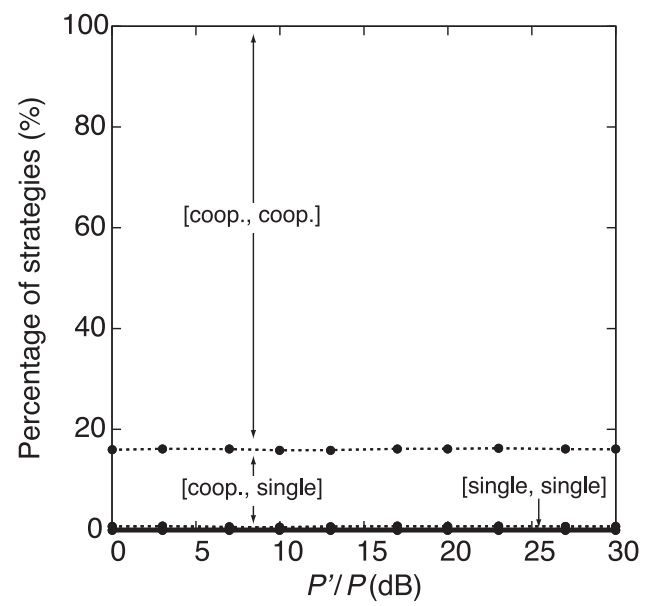

(b) Under equilibrium conditions.

Fig. 7 Percentage of selected strategies of case $(\mathrm{RD}, \mathrm{SR})$. Non-selected strategies are not pointed out in the graph.

network capacity. However, the equilibrium BE is saturated under high transmit power conditions and the difference between the equilibrium BE and the network capacity increases with the transmit power. This is because in the DARSS game both selfish sources tend to transmit, as shown in Fig. 7(b), and consequently the equilibrium BE is limited by the interference.

In contrast to the network capacity, the equilibrium BE's of case $_{(\mathrm{RD}, \mathrm{R})}$ and case (no coop.) outperform those of $\operatorname{case}_{(\mathrm{RD}, \mathrm{SR})}$ and case $(\mathrm{R}, \mathrm{SR})$ when the transmit power is high. The reason for this is as follows. As shown in Fig. 7(b), when each source can select the (RD, SR) scheme and the non-cooperative schemes, cooperative relaying is often selected as a relaying scheme under the equilibrium condition. A similar result applies to case $(\mathrm{R}, \mathrm{SR})$. In addition, when the transmit power is high, the interference has a more significant impact on the received SINR, as compared to the noise. Therefore, the equilibrium BE's of case $_{(\mathrm{RD}, \mathrm{SR})}$ and $\operatorname{case}_{(\mathrm{R}, \mathrm{SR})}$ are less than those of case $\mathrm{RD}_{(\mathrm{RD}, \mathrm{R})}$ and case (no coop.) $_{\text {. }}$, as shown in Sect. 3.4.

Figure 8 shows the $1 \%$ total outage $\mathrm{BE}$ for the networks

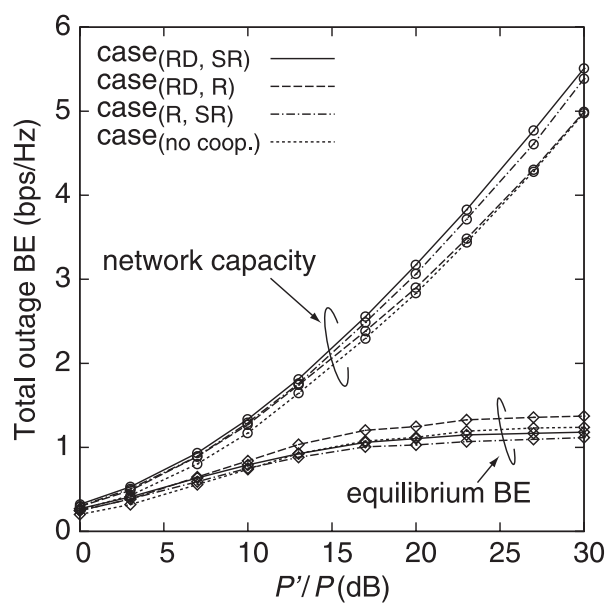

Fig. 8 Transmit power dependence of total outage BE.

with at least one Nash equilibrium. The introduction of cooperative relaying always increases the network capacity, and it also increases the equilibrium BE when the transmit power is low. Note that, in contrast to Fig. 3(b), the equilibrium BE's of case $e_{(R D, S R)}$ and case $e_{(R, S R)}$ do not always outperform those of case $(\mathrm{RD}, \mathrm{R})$ and case (no coop.) . This is because two source-destination pairs can be closely located in the DARSS game unlike the assumptions in the theoretical analysis. Because the received SINR of (RD, SR) and (R, $\mathrm{SR}$ ) are more significantly affected by the interference than that of $(\mathrm{RD}, \mathrm{R})$ and non-cooperative schemes, if two sourcedestination pairs are closely located and transmit power is high, (RD, SR) and (R, SR) do not achieve high gain compared in the theoretical analysis.

While we assume the two-player game, the equilibrium $\mathrm{BE}$ in N-player game will be lower than the network capacity as in the two-player game. This is because the strategy that maximize the $\mathrm{BE}$ of each communication pair does not necessarily correspond to the strategy that maximize the total BE regardless of the number of players. Note that, in the $\mathrm{N}$-player game, Nash equilibrium can be calculated through (29) instead of (30).

\section{Conclusion}

In the present paper, the performance of cooperative relaying networks with ARSS was analyzed. First, we calculated the outage BE in the interference-limited environments. Numerical results revealed that the introduction of cooperative relaying can improve the outage BE. Next, we evaluated the total BE under centralized control and decentralized control. In order to evaluate these performance, we defined the DARSS game. The simulation results show that the introduction of cooperative relaying with the centralized control always increases the network capacity. In addition, the simulation results also show that when each source independently selects the relaying scheme to maximize its own BE, the introduction of cooperative relaying, such as (RD, SR), in which the source and relay station transmit simultane- 
ously may reduce the total $\mathrm{BE}$ because of the increase in the number of interference signals. In this case, cooperative relaying, such as (RD, R), in which the number of interference signals does not increase, should be used.

\section{Acknowledgments}

The present study was supported in part by a Grant-in-Aid for Scientific Research (A) (no. 20246067) from the Japan Society for the Promotion of Science (JSPS).

\section{References}

[1] A. Sendonaris, E. Erkip, and B. Aazhang, "User cooperation diversity-part I: System description,” IEEE Trans. Commun., vol.51, no.11, pp.1927-1938, Nov. 2003.

[2] A. Sendonaris, E. Erkip, and B. Aazhang, "User cooperation diversity-part II: Implementation aspects and performance analysis," IEEE Trans. Commun., vol.51, no.11, pp.1939-1948, Nov. 2003.

[3] J.N. Laneman, D.N.C. Tse, and G.W. Wornell, "Cooperative diversity in wireless networks: Efficient protocols and outage behavior," IEEE Trans. Inf. Theory, vol.50, no.12, pp.3062-3080, Dec. 2004.

[4] R.U. Nabar, H. Bölcskei, and F.W. Kneübuhler, "Fading relay channels: Performance limits and space-time signal design,’ IEEE J. Sel. Areas Commun., vol.22, no.6, pp.1099-1109, Aug. 2004.

[5] M. Haenggi, "Analysis and design of diversity schemes for ad hoc wireless networks," IEEE J. Sel. Areas Commun., vol.23, no.1, pp.19-27, Jan. 2005.

[6] T. Ohtsuki, "Performance analysis of statistical STBC cooperative diversity using binary sensors with observation noise," IEICE Trans. Commun., vol.E89-B, no.3, pp.970-973, March 2006.

[7] K. Yamamoto, H. Maruyama, T. Shimizu, H. Murata, and S. Yoshida, "Spectral efficiency of fundamental cooperative relaying in interference-limited environments," IEICE Trans. Commun., vol.E91-B, no.8, pp.2674-2682, Aug. 2008.

[8] A. Wittneben, I. Hammerstrom, and M. Kuhn, "Joint cooperative diversity and scheduling in low mobility wireless networks," Proc. IEEE GLOBCOM, vol.2, pp.780-784, Nov. 2004.

[9] C.U. Saraydar, N.B. Mandayam, and D.J. Goodman, "Efficient power control via pricing in wireless data networks," IEEE Trans. Commun., vol.50, no.2, pp.291-303, Feb. 2002.

[10] A.B. MacKenzie and S.B. Wicker, "Selfish users in Aloha: A gametheoretic approach," Proc. IEEE VTC 2001-Fall, pp.1354-1357, Oct. 2001.

[11] R.U. Nabar and H. Bölcskei, "Space-time signal design for fading relay channels," Proc. IEEE GLOBECOM'03, vol.4, pp.1952-1956, Dec. 2003

[12] S. Toumpis and A.J. Goldsmith, "Capacity regions for wireless ad hoc networks," IEEE Trans. Wireless Commun., vol.2, no.4, pp.736-748, July 2003.

[13] M.S. Alouini and A.J. Goldsmith, "Area spectral efficiency of cellular mobile radio systems," IEEE Trans. Veh. Technol., vol.48, no.4, pp.1047-1066, July 1999.

[14] K. Yamamoto and S. Yoshida, "Tradeoff between area spectral efficiency and end-to-end throughput in rate-adaptive multihop radio networks," IEICE Trans. Commun., vol.E88-B, no.9, pp.3532-3540, Sept. 2005.

[15] J. Jun and M.L. Sichitiu, "Fairness and QoS in multihop wireless networks," Proc. IEEE VTC 2003-Fall, pp.6-9, Oct. 2003

[16] V. Gambiroza, B. Sadeghi, and E.W. Knightly, "End-to-end performance and fairness in multihop wireless backhaul networks," Proc. MOBICOM 2004, pp.287-301, Sept.-Oct. 2004.

[17] E. Anshelevich, A. Dasgupta, E. Tardos, and T. Wexler, "Nearoptimal network design with selfish agents," Proc. Thirty-Fifth Annual ACM Symposium on Theory of Computing, pp.511-520, 2003.
[18] S. Eidenbenz, V.A. Kumar, and S. Zust, "Equilibria in topology control games for ad hoc networks," Proc. DialM-POMC'03, pp.2-11, Sept. 2003.

[19] D. Fudenberg and J. Tirole, Game Theory, MIT Press, 1991.

[20] K. Yamamoto and S. Yoshida, "Game-theoretic approach to capacity and stability evaluations of decentralized adaptive route selections in wireless ad hoc networks," IEICE Trans. Commun., vol.E88-B, no.3, pp.1009-1016, March 2005.

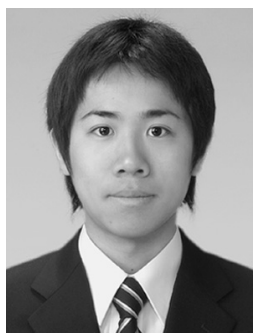

Kunihiko Teshima received the B.E. degree in electrical and electronic engineering from Kyoto University in 2007, and the M.E. degree in informatics from Kyoto University in 2009 In 2009, he joined NTT DOCOMO INC. He received the IEEE VTS Japan 2008 Student Paper Award in 2008, and the 5th IEEE Kansai Student Researcher Encouragement Award in 2009.

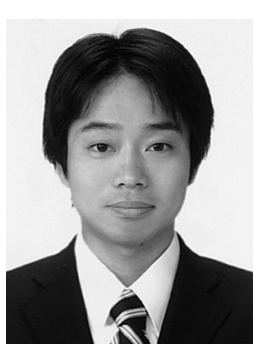

Koji Yamamoto received the B.E. degree in electrical and electronic engineering from Kyoto University in 2002, and the M.E. and Ph.D. degrees in informatics from Kyoto University in 2004 and 2005, respectively. Since 2005, he has been an assistant professor of the Graduate School of Informatics, Kyoto University. From 2008 to 2009 , he was a visiting researcher at Wireless@KTH, Royal Institute of Technology $(\mathrm{KTH})$ in Sweden. His research interests include game theory, spectrum sharing, and cooperative multi-hop networks. He received the PIMRC 2004 Best Student Paper Award in 2004, the Ericsson Young Scientist Award in 2006, and the Young Researcher's Award from the IEICE of Japan in 2008. He is a member of the IEEE.

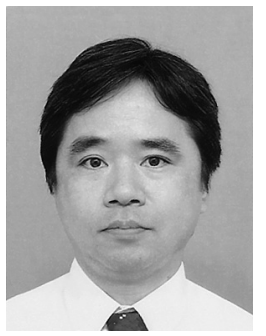

Hidekazu Murata received the B.E., M.E., and $\mathrm{Ph} . \mathrm{D}$. degrees in electronic engineering from Kyoto University, Kyoto, Japan, in 1991, 1993, and 2000, respectively. In 1993, he joined the Faculty of Engineering, Kyoto University. From 2002 to 2006, he was an Associate Professor of Tokyo Institute of Technology. He has been at Kyoto University since October 2006 and is currently an Associate Professor of Department of Communications and Computer Engineering, Graduate School of Informatics. His major research interests include signal processing and its hardware implementation, with particular application to cooperative wireless networks with cognitive radio capabilities. He received the Young Researcher's Award from the IEICE of Japan in 1997, the Ericsson Young Scientist Award in 2000, and the Young Scientists' Prize of the Commendation for Science and Technology by the Minister of Education, Culture, Sports, Science and Technology in 2006. He is a member of the IEEE and SITA. 


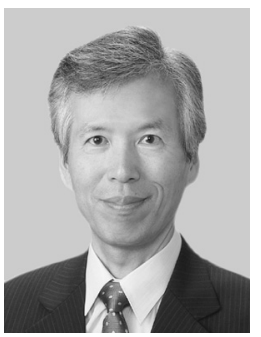

Susumu Yoshida received the B.E., M.E. and $\mathrm{Ph} . \mathrm{D}$. degrees all in electrical engineering from Kyoto University, Kyoto, Japan in 1971, 1973 and 1978, respectively. Since 1973, he has been with the Faculty of Engineering, Kyoto University and currently he is a full professor of the Graduate School of Informatics, Kyoto University. During the last 30 years, he has been mainly engaged in the research of wireless personal communications. His current research interest includes highly spectrally efficient wireless transmission techniques and distributed controlled wireless networks. During 1990-1991, he was a visiting scholar at WINLAB, Rutgers University, U.S.A. and Carleton University in Ottawa, Canada. He served as a TPC Chair of IEEE VTC 2000-Spring, Tokyo. He was a guest editor of IEEE J-SAC on Wireless Local Communications published in April and May 1996. He received the IEICE Achievement Award and Ericsson Telecommunication Award in 1993 and 2007, respectively. 